\section{Intrapericardial Infusion of Urokinase for the Treatment of Purulent Pericarditis}

Key words: fibrinolytic therapy, intrapericardial catheter, peri-
cardiectomy

Intrapleural fibrinolytic therapy has become common practice, but reports of intrapericardial use are quite limited. We report a case in which transcatheter infusion of urokinase successfully resulted in the drainage of coagulated pericardial effusion.

A 41-year-old man noticed right supraclavicular lymphadenopathy in July 1997. He was diagnosed as having pulmonary tuberculosis and tuberculous lymphadenitis of both mediastinal and right supraclavicular lesions at his previous hospital. Despite administration of an anti-tuberculosis drug course, right supraclavicular lymphadenopathy became enlarged in February 1998 and ruptured spontaneously one month later. The patient left the deep skin fistula unsterilized for two weeks, and subsequently developed high fever and dyspnea and was brought to our institute. Chest computed tomography showed bilateral infiltration of the upper lung field, massive pericardial and pleural effusion, marked swelling of the mediastinal lymph nodes and wide anterior mediastinal soft tissue density. A central vein catheter ( $16 \mathrm{Fr}$ ) with a single lumen was inserted for pericardial effusion through the subxiphoid approach. Pericardial and pleural effusion revealed a high adenosine deaminase titer and a positive polymerase chain reaction for tuberculous bacilli. The patient's condition was interpreted as the development of tuberculous mediastinitis, pleuritis, pericarditis and concurrent bacterial infection of a skin fistula, with the infection possibly invading the upper mediastinum. Levofloxacin was added to the anti-tuberculous regimen and cefmetazole sodium was started for skin and mediastinal infection. After draining $800 \mathrm{ml}$ of pericardial effusion, the indwelling catheter stopped producing fluid on the 4th day. A small amount of pus was collected in the catheter and revealed a positive culture for $\alpha$-Streptococcus. Echocardiography revealed 10-mm thick residual pericardial effusion and disappearance of the pendular motion of the heart in accordance with cardiac contraction within the fluid, which was observed at the initial examination. Secondary bacterial pericarditis through the catheter with resultant coagulation of fibrinous effusion was diagnosed and intrapericardial infusion of urokinase 120,000 U diluted with $10 \mathrm{ml}$ of saline was conducted five times with the informed consent of the patient. Urokinase was retained intrapericardially for 12 hours for each administration, then suction with a syringe was performed manually. Each of the first two procedures successfully removed $250 \mathrm{ml}$ of bloody gelatinous pus. Each of the last three procedures resulted in the removal of 300 to $500 \mathrm{ml}$ of serous bloody fluid with a simultaneous decrease in right pleural effusion. Fistula formation between the pericardial and the right pleural cavity was suspected and administration of urokinase was withheld. The coagulation profile did not change significantly before and after the course of intrapericardial urokinase infusion. Vital signs were stable and no arrhythmia was recorded during the procedures. The patient's condition was stabilized after complete drainage of pleural effusion and a 35-day course of cefmetazole. The intrapericardial catheter was withdrawn after daily rinsing of the cavity with bronopol for three weeks. The patient was discharged after resection of the intractable skin fistula, without evidence of diastolic disturbance by echocardiography. $\mathrm{He}$ has been well for three years following his discharge.

Subxiphoid drainage with a catheter is common practice for pericardial effusion, but loculation or coagulation of fluid may disturb drainage and cause subsequent sequelae. Subxiphoid pericardial irrigation with streptokinase and streptokinase-dornase was first documented in 1951 (1) for purulent pericarditis. Despite its effectiveness, the procedure was almost forgotten and surgical drainage and pericardiectomy have been the mainstay of therapy for this condition (2). The efficacy of intrapericardial fibrinolytics was rediscovered by Bennett in 1984 (3) and thereafter, several reports documented its usefulness in draining the fibrinous pericardial fluid $(4,5)$. Intrapericardial use of urokinase, which is superior to streptokinase due to its lack of allergic potency (4), was attempted by Winkler et al and the dosage used ranged from $200,000 \mathrm{U}$ to $2,200,000 \mathrm{U}$ (4). In our case, $120,000 \mathrm{U}$ was sufficient to dissolve the fibrinous material, and an adequate dosage should be determined in future studies.

The findings in the present case indicate that intrapericardial infusion of urokinase is a safe and effective method for draining coagulated pericardial effusion and should be considered before using the surgical approach.

\section{Mitsuhiro Kamimura, Tsuneo Suzuki and Koichiro Kudo}

The Department of Pulmonology, The International Medical Center of Japan, Tokyo

Received for publication September 14, 2001; Accepted for publication January 28,2002

Reprint requests should be addressed to Dr. Mitsuhiro Kamimura, the Department of Pulmonology, The International Medical Center of Japan, 1-21-1 Toyama-cho, Shinjuku-ku, Tokyo 162-8655

\section{References}

1) Wright LT, Smith DH, Rothman M, Metzger WI, Quash ET. Use of streptokinase-dornase in certain surgical conditions. J Int Coll Surg 15: 286298, 1951.

2) Majid AA, Omar A. Diagnosis and management of purulent pericarditis. Experience with pericardiectomy. J Thorac Cardiovasc Surg 102: 413417, 1991.

3) Bennett EV. Purulent pericarditis. J Thorac Cardiovasc Surg 87: 641- 
$642,1984$.

4) Winkler WB, Karnik R, Slany J. Treatment of exudative fibrinous pericarditis with intrapericardial urokinase. Lancet 344: 1541-1542, 1994.
5) Mann-Segal DDM, Shanahan EA, Jones B, Ramasamy D. Purulent pericarditis. Rediscovery of an old remedy. J Thorac Cardiovasc Surg 111: 487-488, 1996. 